Research Article

\title{
In Silico Prediction of the Mode of Action of Viola odorata in Diabetes
}

\author{
Manal Ali Buabeid $\left(\mathbb{D},{ }^{1}\right.$ El-Shaimaa A. Arafa $\mathbb{D D}^{1,2}$ Waseem Hassan $(\mathbb{D})^{3}$ \\ and Ghulam Murtaza iD ${ }^{3}$ \\ ${ }^{1}$ College of Pharmacy and Health Sciences, Ajman University, Ajman 346, UAE \\ ${ }^{2}$ Department of Pharmacology and Toxicology, Faculty of Pharmacy, Beni-Suef University, Beni Suef 62514, Egypt \\ ${ }^{3}$ Department of Pharmacy, COMSATS University Islamabad, Lahore Campus, 54000, Pakistan
}

Correspondence should be addressed to Manal Ali Buabeid; m.buabeid@ajman.ac.ae

Received 16 April 2020; Revised 27 June 2020; Accepted 5 October 2020; Published 31 October 2020

Academic Editor: K. H. Mok

Copyright (c) 2020 Manal Ali Buabeid et al. This is an open access article distributed under the Creative Commons Attribution License, which permits unrestricted use, distribution, and reproduction in any medium, provided the original work is properly cited.

\begin{abstract}
Background. The metabolic syndrome increases the risk of different diseases such as type 2 diabetes. The prevalence of metabolic syndrome has rapidly grown and affected more than 230 million people worldwide. Viola odorata is a traditionally used plant for the treatment of diabetes; however, its mechanism to manage diabetes is still unknown. Purpose. This study was designed to systematically assess the mechanism of action of Viola odorata in diabetes. Methods. An extensive literature search was made to establish an ingredient-target database of Viola odorata. Of these, targets related to diabetes were identified and used to develop a protein-protein interaction network (PPIN) by utilizing the STITCH database. The obtained PPIN was assessed through Gene Ontology (GO) enrichment analysis based on ClueGO plugin. Results. According to the acquired data, there were about 143 chemical constituents present in Viola odorata having 119 protein targets. Of these, 31 targets were established to give the pharmacological effect against diabetes. The UniProt database was used for screening of 31 targets, out of which Homo sapiens contained 22 targets. Ultimately, 207 GO terms, grouped into 41 clusters, were found by gene analysis, and most of them were found to be linked with diabetes. According to findings, several proteins including TP53, BCL2, CDKN1A, 1L6, CCND1, CDKN2A, and RB1 have a significant role in the treatment of diabetes by Viola odorata. Conclusion. The possible activity of Viola odorata in the management of diabetes may be mediated by several molecular mechanisms, including the glutamine metabolic process, IRE1-mediated unfolded protein response, and pentose metabolic process.
\end{abstract}

\section{Introduction}

Metabolic syndrome is a group of interlinked diseases that occur together, for instance, hypertension and hyperglycemia, and leads to increased risk of type 2 diabetes. The prevalence of metabolic syndrome has rapidly grown. To date, it has affected more than 230 million people worldwide [1]. Obesity is one of the major contributing factors in producing metabolic syndrome. The major drawback of obesity is noninsulin-dependent diabetes mellitus and cardiovascular diseases [2]. Diabetes is a metabolic disorder categorized by hyperglycemia resulting from defects in insulin secretion, insulin action, or both [3]. According to a report based on death certificates conducted in 2010, it is the sixth leading cause of death in the US. In Pakistan, this disease is more prevalent in the urban population (11.1\%), as compared to rural $(9.4 \%)$ [4].

The medicines clinically used for the treatment of diabetes have many adverse effects, and over time, half of the drugs have become less responsive to a metabolic disorder. Thus, alternative therapy always remains the second option to treat diseases like metabolic disorder. It has already been reported that the Viola odorata plant is traditionally used to treat diabetes [5]. Viola odorata Linn. is a medicinal plant that belongs to the family Violaceae [5]. It is commonly known as sweet violet and found in Europe and Asia [6]. 
Viola odorata has been reported to contain phenolics, flavonoids, tannins, proteins, saponins, and alkaloids [7]. The active compounds present in Viola include salicylic acid, anthocyanins, butyl-2-ethylhexyl phthalate, and 5,6,7,7a-tetrahydro-4,4,7a-trimethyl-2(4H)-benzofuranone. Other active compounds of Viola odorata are cyclotides such as cycloviolacin O1, which are responsible for the antidiabetic potential of this plant. Approximately, 30 cyclotides have been recognized to date. Although there are many studies related to the antimetabolic effect of Viola odorata $[5,7,8]$, there is no study that discusses the mechanism of action of Viola odorata as an antidiabetic agent.

Besides the "one drug-one target" model of drug discovery, "multiple drugs-multiple targets" concept, also known as polypharmacology, is widely spreading, especially in the field of multitargeted natural products and their mixtures including various nutraceuticals and traditional Chinese medicines (TCMs). This paradigm is promisingly elaborated by network pharmacology, which is a rapidly growing concept comprising integrated knowledge of polypharmacology and system biology [9]. Network pharmacology plays two essential roles in drug development. Firstly, it contributes to establishing a logical network model and predicting the drug targets based on the literature or publicly available databases. Secondly, the mode of drug action can be explored for the network equilibrium principle [10]. Network pharmacology is useful for developing a network comprising chemical ingredient-protein-disease and acquiring vital information about network protein regulation, which facilitates the drug discovery process through the knowledge of genome project and Gene Ontology (GO) [11-13]. In network pharmacology, molecular docking plays an essential role in the investigation of the mode of action of therapeutic moieties, particularly a multi-ingredient regimen [12].

The present study described the use of network pharmacology including molecular docking to predict the mode underlying the antidiabetic effects of Viola odorata. In this approach, chemical ingredients of Viola odorata were searched, ingredient-related protein targets were identified, and core functions were recognized through the proteinprotein interaction network and molecular docking. To our knowledge, the mode of action of Viola odorata has not yet been studied by a network pharmacology approach; therefore, our approach offers a potential strategy to explore the mode of Viola odorata against diabetes.

\section{Methodology}

In the present era, computational methods have become more useful to compile data related to drug discovery and for the identification of drug targets. The mechanism of Viola odorata against diabetes can be described by using the STITCH database along with network development and its investigation. This network development approach was aimed at finding out the multitarget compounds (Figure 1). At first, the chemical components of Viola odorata and their protein targets in Homo sapiens were defined. Secondly, a network was created called the Viola odorata-target interaction network, which was further assessed by different Gene
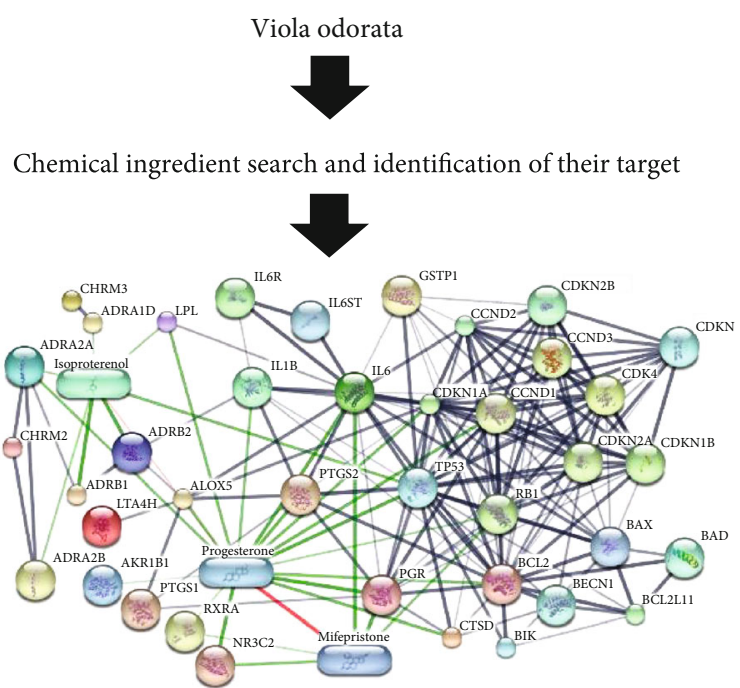

Protein interaction network developed via STITCH

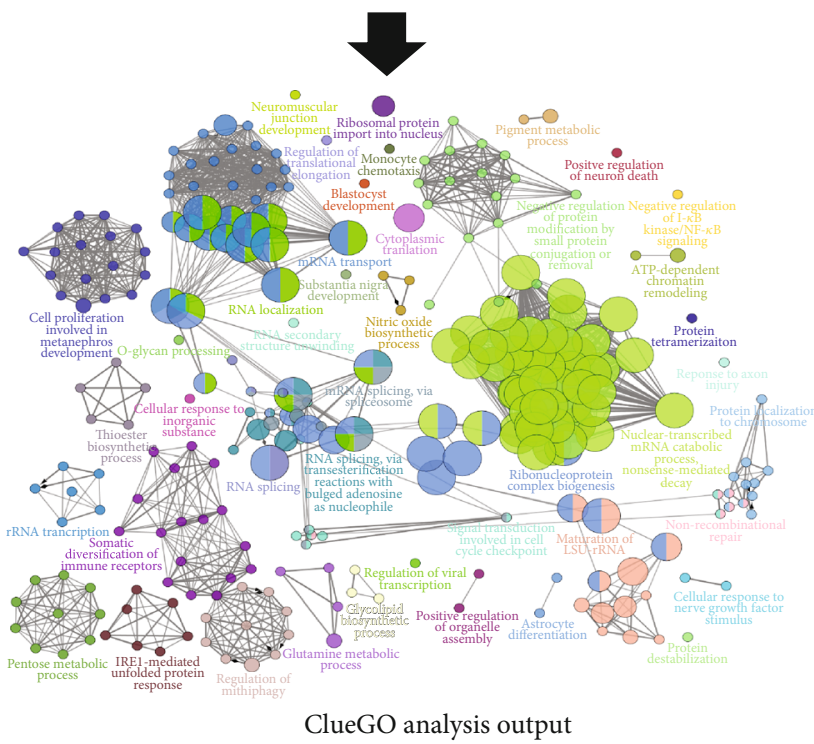

FIgure 1: Flowchart demonstration of steps carried out in this systematic study.

Ontology (GO) terms. The molecular mechanism of Viola odorata against diabetes was explored through GO enrichment analysis and biological assessment utilizing Cytoscape and its plugin, ClueGO.

2.1. Recovery of Chemical Constituents and Their Targets. Viola odorata was searched for its chemical constituents using literature [12-20] as well as the Cybase database (http://www.cybase.org.au/index.php, accessed in November 2019). After retrieving the protein targets of these constituents, UniProtKB (http://www.uniprot.org, accessed in November 2019) was used for the extraction of gene names. To identify their association with diabetes, these molecular targets were examined based on the Kyoto Encyclopedia of Genes and Genomes (KEGG, http://www.kegg.jp/, accessed in November 2019). 
2.2. Network Construction and Its Analysis. The mode of action of Viola odorata was described systematically by analyzing the interaction between identified targets by using the STITCH 5.0 database (http://stitch.embl.de/). This database gave us the statistics of predicted and known protein interactions, which were classified into direct and indirect interactions. The sources to find out the information of these interactions were high-throughput experimental findings, coexpression, genome studies, and text mining. The STITCH 5.0 database technique comprised the data of 9.6 million proteins extracted from approximately 2300 organisms. Then, the analysis of the association between the chemical ingredient and the identified protein targets was done by using the interaction network to assess the mode of action of Viola odorata and its pharmacodynamic components.

2.3. GO Enrichment Analysis. For dissection of the target genes in a hierarchically structured mode, GO enrichment analysis was introduced to identify and assess the specific biological properties of the potential targets. This approach is reasonably promising for the investigation of the mode of action of Viola odorata against diabetes. Cytoscape 3.4 .0 software and its plugin ClueGO were used to develop, visualize, and evaluate the protein target network and assess the biological pathways to find out the mode of action of Viola odorata and its pharmacodynamic features $[22,23]$. The $p$ value threshold was set at 0.05 for ClueGO analysis. Additionally, this study involved the use of a medium network type option and a two-sided hypergeometric test with the Bonferroni correction. In comparison to the global and the detailed type of networks, the medium network shows GO terms originated in the level of 4-8, with a medium number of genes associated and a medium percentage of uploaded genes found. Lastly, an organic layout algorithm was used to visualize the functional network.

2.4. Structure Preparation and Molecular Docking. A wide array of compounds such quercetin, alrestatin, eugenol, coumarin, limonene, shikimic acid, stigmasterol, and rutin are present in Viola odorata. The traditional Chinese medicine systems pharmacology database (http://lsp.nwsuaf.edu $. c n / t c m s p . p h p)$ revealed that tumor necrosis factor (TNF) and aldose reductase (AR) are the main targets of these compounds.

3D structures of selected compounds eugenol, coumarin, limonene, shikimic acid, stigmasterol, and rutin (Figure 2) were constructed by the SYBYL-X 1.3/SKETCH module [24] followed by energy minimization according to the Tripos force field with the Gasteiger-Hücke atomic charge [24]. The cocrystallized structures of tumor necrosis factor (TNF) and aldose reductase (AR) were downloaded from RCSB Protein Data Bank (PDB ID: 2AZ5 [25] and 4GCA [26], respectively) to be regarded as starting points for molecular docking studies. The obtained 3D structures of 2AZ5 and 4GCA were carefully analyzed to authenticate the chemical accuracy using structure preparation tools in the biopolymer module of SYBYL-X 1.3 [5]. Missing hydrogen atoms were computationally added. Atom types were assigned appropriately, and atomic charges were applied according to the AMBER $7 \mathrm{ff} 99$ force field. The SYBYL-X 1.3/protein preparation module [1] was then used to pick sensible protonation states for titratable residues, followed by energy minimization of each protein to avoid steric clashes using the Powell algorithm with a convergence gradient of $0.5 \mathrm{kcal} /(\mathrm{mol} . \AA)$ for 1000 cycles, while keeping backbone atoms fixed. In order to investigate the binding modes of the selected compounds in the active site of proteins chosen, flexible molecular docking simulations were performed using the Surflex-Dock module of the molecular modeling software package SYBYL-X 1.3 [24] by adopting the same protocol and parameters as reported in our previous publications $[27,28]$. For each ligand-receptor complex system, at least 20 best-docked poses were conclusively saved. The best putative poses of ligands were graded by adopting the Hammerhead scoring function (Cscore) [29]. Furthermore, the most frequently used TNF and AR inhibitors quercetin [30-34] and alrestatin [35-37], respectively, were used as benchmarks to compare docking poses of our tested compounds with those of standard compounds. Therefore, the standard compounds quercetin and alrestatin were also docked into corresponding target proteins $\mathrm{TNF}$ and $\mathrm{AR}$, respectively, using the aforementioned protocol and parameters.

\section{Results}

3.1. Recovery of Chemical Ingredients and Their Targets. This work involved the retrieval of 143 chemical constituents of Viola odorata through a literature search (Supplementary Table 1). The features of these ingredients comply with the definition of a drug. Studies from different kinds of the literature confirmed that these chemical constituents had around 119 protein targets (Supplementary Table 2). The literature study reveals that Viola odorata is a useful alternative therapy for diabetes. Accordingly, out of 119, 31 targets of Viola odorata were found to exert pharmacological influence against diabetes. The UniProt database mapping (http://www.uniprot.org/) was used for the standardization of the identified 31 protein targets. Out of these 31 targets, 22 proteins were present in Homo sapiens.

3.2. Network Construction and Its Analysis. Twenty-two target proteins were present in the STITCH-based proteinprotein interaction network (PPIN) (accessed in Nov 2019) (Figure 3). This PPIN was constructed using a medium probabilistic confidence score, i.e., 0.400 . The network comprised 42 nodes and 152 edges. Network nodes act as the representatives of the protein targets or their relevant genes. The lines connecting various nodes indicate the interaction among the corresponding genes. There were 20 functional interactions out of 42 interactions. According to network statistics, the $p$ value of PPIN enrichment was 0.001 , which is a significantly very small value. It exhibits that there is a significant number of the observed edges, as well as the found nodes, which are not random. In the case of a random selection of nodes, the expected number of edges for this PPIN was 54 . The average node degree of PPIN was 7.79, while its average clustering coefficient value was 0.749 . In PPIN, the average number of interactions of protein targets at the threshold score is 


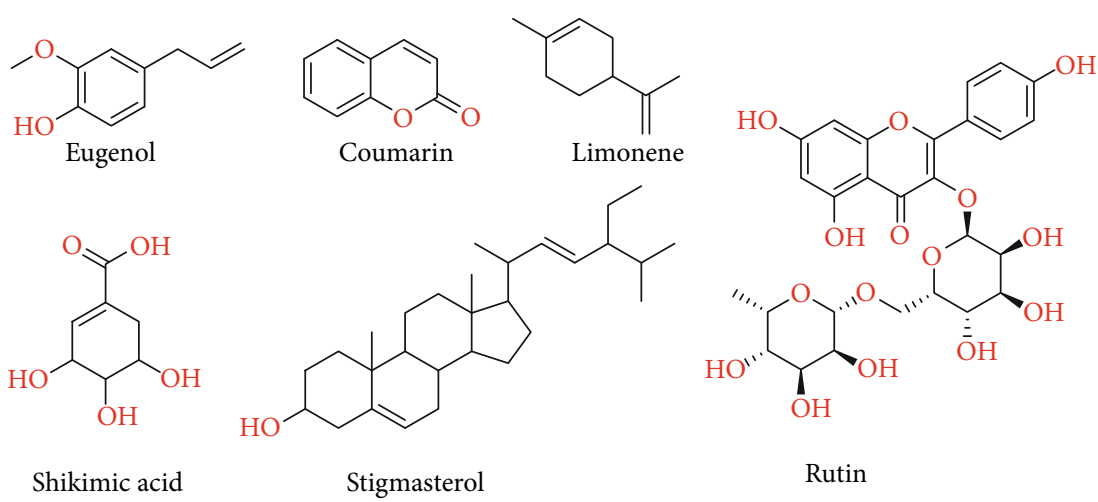

FIgURE 2: Chemical structures of the selected phytoconstituents for the molecular docking study.

represented by the average node degree. Alternatively, the degree of connectivity of nodes in a PPIN is called the clustering coefficient. The connectivity of the network increases with the increased value of the clustering coefficient.

Moreover, 19 hubs were found in the PPIN. The node degree is a quantitative property of a node that can be explained as the number of interactions of nodes in a network. A node is known as a hub if its number of linkages is higher than the average node degree $=7.79$. The findings revealed that BCL2 and TP53 proteins hold the highest node degree of the node, which is 23. Based on the node degree, IL6, CDKNIA, and progesterone were the subsequent proteins that had a degree of 18 . Table 1 shows the degree of each node. It has already been documented that diabetes has the involvement of these protein targets (in particular, the hubs and the functional nodes) such as TP53, BCL2, and CDKN1A. Table 2 gives the action view of functional nodes present in PPIN, revealing that the activation of functional proteins except BIK, BAD, CCND1, and BCL2L11 could be done by Viola odorata. On the other hand, Viola odorata is involved in the inhibition of BIK, BECN1, IL6R, and BCL2L11. The results also describe the binding potential of Viola odorata ingredients with all identified functional proteins. Table 2 also represents that Viola odorata is involved in many other functions such as cellular reactions, catalysis, posttranslational modifications, and gene expression.

3.3. GO Enrichment Analysis. ClueGO-mediated enrichment analysis was carried out for the identified protein targets of Viola odorata by using GO terms to explain their biological activities. As a result of this GO enrichment analysis, 41 GO terms associated with 207 genes were obtained in the form of small clusters, including the glutamine metabolic process, IRE1-mediated unfolded protein response, and pentose metabolic process (Supplementary Table 3, Figure 4). Further advancement on the mechanism of action of Viola odorata can be made based on the acquired findings.

3.4. Molecular Docking. In the present work, all six bioactive phytoconstituents were individually docked into the active site of TNF and AR proteins using the Surflex-Dock module of SYBYL-X 1.3. [38]. The docking scores (Cscore) of eugenol, coumarin, limonene, shikimic acid, stigmasterol, and rutin for TNF are 4.60, 2.03, 3.95, 2.58, 5.14, and 6.29, respectively, which indicates that rutin and stigmasterol exhibit substantial binding affinities towards TNF. The docking comparison with the standard compound has revealed that rutin and stigmasterol (Cscore $=6.29$ and 5.14, respectively) share similar binding affinity towards TNF as that observed in the quercetin-TNF system $($ Cscore $=6.06)$. However, eugenol, coumarin, limonene, and shikimic acid displayed moderate to weak binding affinity towards TNF. Moreover, the docking of all six compounds along with standard compound alrestatin in the active site of $\mathrm{AR}$ protein has revealed that rutin and eugenol are more strongly bonded to $\mathrm{AR}$ (Cscore $=6.87$ and 5.13, respectively) than alrestatin $($ Cscore $=4.94)$, whereas coumarin-, limonene-, shikimic acid-, and stigmasterol-bonded systems (Cscore $=3.41$, $4.17,2.04$, and 4.58 , respectively) have demonstrated moderate to weak binding affinity as compared to the alrestatin-AR system. As demonstrated by docking scores, rutin exhibits the highest binding affinity towards TNF and AR proteins among all selected and standard compounds, while shikimic acid has shown a nonsignificant binding affinity towards both proteins. However, the superior binding affinity of rutin to standard compounds towards TNF and AR might be attributed to its mighty structure substituted with various $\mathrm{H}$-bond donor/acceptor features. For further details, corresponding docking scores and key residues involved in $\mathrm{H}$-bonding are summarized in Supplementary Table 4.

To inspect the molecular features responsible for variations in binding affinity of selected ligands towards TNF, the top-ranked docking conformations for all six ligands in their respective complexes were saved. Docking results show that all ligands occupy the same cavity located between the subunits of the TNF dimer. As shown in Figures 5(b)-5(g), all ligands were able to establish at least a couple of $\mathrm{H}$-bond interaction with the TNF dimer with the exception of limonene, which showed no H-bond interaction due to lack of any het-atom in its structure. Ideally, TNF-inhibitor interactions are hydrophobic and shape-driven, as the inhibitor structure needs to be large enough to interact with both subunits and to prevent binding of the third subunit to the TNF dimer [39]. Since nonpolar residues, i.e., Gly, Leu, and Tyr, are predominant in the binding site of the 

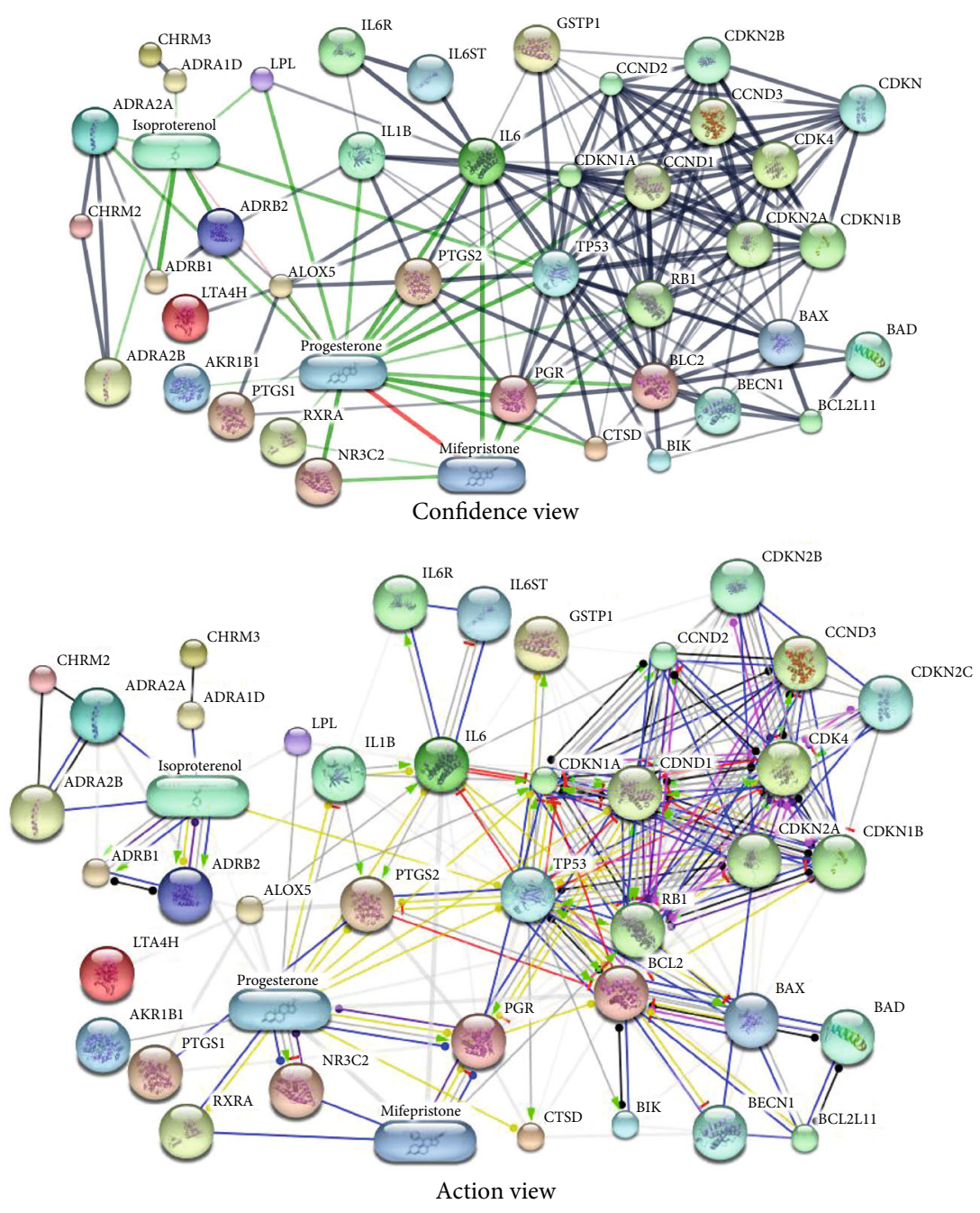

Figure 3: Protein-protein interaction network of diabetes-related protein targets of Viola odorata. In a confidence view, the nodes represent proteins. This network contains only colored nodes (no white node), indicating that the query proteins belong to the first shell of interactors only. Small nodes indicate proteins of unidentified 3D structure, while Large nodes reflect proteins with known 3D structure. The edges in this network represent protein-protein interactions, which are intended to be characteristic and expressive; i.e., proteins together play their role in a shared function; this does not essentially indicate that there is a physical attachment among them. Thick lines reflect stronger associations, while grey and green lines represent the protein-protein and chemical-protein associations, respectively. In an action view, the action type is shown by colored edges as follows: Activation, Inhibition, Binding, Catalysis, Phenotype, Posttranslational modification, Reaction, and Transcriptional regulation. Action effects are indicated by the following signs: Positive, Negative, and Unspecified. Note: LTA4H: leukotriene A4 hydrolase; CTSD: cathepsin D; CHRM3: cholinergic receptor, muscarinic 3; CDK4: cyclin-dependent kinase 4; IL6: interleukin 6 (interferon, beta 2); IL1B: interleukin 1, beta; ADRA2A: adrenoceptor alpha 2A; AKR1B1: aldo-keto reductase family 1, member B1 (aldose reductase); ADRB2: adrenoceptor beta 2, surface; LPL: lipoprotein lipase; CHRM2: cholinergic receptor, muscarinic 2; PGR: progesterone receptor; BCL2: B-cell CLL/lymphoma 2; NR3C2: nuclear receptor subfamily 3, group C, member 2; PTGS1: prostaglandin-endoperoxide synthase 1; PTGS2: prostaglandin-endoperoxide synthase 2; ADRB1: adrenoceptor beta 1; ALOX5: arachidonate 5-lipoxygenase; ADRA1D: adrenoceptor alpha 1D; GSTP1: glutathione S-transferase pi 1; ADRA2B: adrenoceptor alpha 2B; RXRA: retinoid X receptor, alpha; CCND1: cyclin D1; CCND3: cyclin D3; CDKN2A: cyclin-dependent kinase inhibitor 2A; RB1: retinoblastoma 1; CDKN1B: cyclin-dependent kinase inhibitor 1B; CDKN1A: cyclin-dependent kinase inhibitor 1A; IL6R: interleukin 6 receptor; BCL2L11: BCL2-like 11; CCND2: cyclin D2; CDKN2B: cyclin-dependent kinase inhibitor 2B (p15, which inhibits CDK4); BAD: BCL2-associated agonist of cell death; BECN1: beclin 1, autophagy related; CDKN2C: cyclin-dependent kinase inhibitor 2C; TP53: tumor protein p53; BIK: BCL2-interacting killer; IL6ST: interleukin 6 signal transducer; BAX: BCL2-associated X protein.

TNF dimer, it may explain good to moderate binding affinity of stigmasterol, eugenol, and limonene towards TNF (Figures 5(b), 5(d), and 5(f)). As shown in Supplementary Table 4, rutin forms the strongest complex with TNF as compared to all studied ligands. Graphical analysis of the rutin-TNF complex has demonstrated that the compound established six H-bond interactions with surrounding residues in a donor acceptor manner (Supplementary Table 4). Moreover, rutin exhibits large enough structure to interact with both subunits of the TNF dimer, which may be attributed to its higher binding affinity towards TNF than other studied complexes. 
TABLE 1: Node degree of diabetes-related protein targets of Viola odorata retrieved via STITCH.

\begin{tabular}{lccccc}
\hline Targets & Degree & Targets & Degree & Targets & Degree \\
\hline LTA4H & 1 & PTGS1 & 3 & IL6R & 3 \\
CTSD & 6 & PTGS2 & 11 & BCL2L11 & 6 \\
CHRM3 & 1 & ADRB1 & 3 & CCND2 & 14 \\
CDK4 & 14 & ALOX5 & 7 & CDKN2B & 12 \\
IL6 & 18 & ADRA1D & 2 & Isoproterenol & 8 \\
IL1B & 8 & GSTP1 & 8 & BAD & 3 \\
ADRA2A & 5 & ADRA2B & 3 & BECN1 & 6 \\
AKR1B1 & 1 & RXRA & 2 & CDKN2C & 11 \\
ADRB2 & 4 & CCND1 & 17 & TP53 & 23 \\
LPL & 3 & CCND3 & 11 & BIK & 4 \\
CHRM2 & 2 & CDKN2A & 16 & IL6ST & 2 \\
PGR & 15 & RB1 & 16 & Progesterone & 18 \\
BCL2 & 23 & CDKN1B & 15 & BAX & 13 \\
NR3C2 & 1 & CDKN1A & 18 & Mifepristone & 6 \\
\hline
\end{tabular}

Conversely, the weakest binding affinity of coumarin and shikimic acid might be attributed to their smaller structure, which may not allow these structures to interact with both subunits of the TNF dimer. Analysis of quercetin-TNF docking pose reveals that the standard compound occupies the same cavity to establish at least six $\mathrm{H}$-bond interactions with common residues as observed in the rutin-TNF complex (Supplementary Figures 1A and 1C). Superimposition of all docked complexes over the standard-TNF complex reveals that all ligands settled in the same binding site of TNF to establish similar pattern interactions.

From Supplementary Table 4 and Figures 6(a)-6(f), out of the six compounds, rutin exhibited a high binding score and interaction towards AR. In the case of the rutin-AR complex (Figure 6(d)), the ligand interacted with the protein by forming seven hydrogen bonds, two $\pi-\pi$ interactions, and several hydrophobic contacts. The two aromatic rings of rutin are firmly anchored in the anionic subsite by the formation of $\pi-\pi$ interactions with $\operatorname{Trp} 20$ and $\operatorname{Trp} 219$. Furthermore, it forms hydrogen bonds with Trp20, Val47, Tyr48, Gln49, Trp111, and Ala299. In addition, residues Trp111, Phe122, Gln183, Pro218, and Leu300 are part of the specificity pocket to establish several hydrophobic and van der Waals (vdW) contacts.

Interestingly, these residues have previously been shown to be responsible for the selective ligand-protein complex formation [26]. Eugenol and stigmasterol have shown the second and third highest docking scores for AR as compared to the AR complex formation (Figures 6(a) and 6(f)). Although eugenol establishes only one $\mathrm{H}$-bond interaction with Leu300, the aromatic ring of eugenol is packed between the top and bottom aromatic rings of residues Trp111 and Tyr309 to create $\pi-\pi$ interactions with these residues. However, these additional $\pi-\pi$ interactions were not observed in the stigmasterol-Ar complex, which may explain slightly higher docking scores for the eugenol-AR system. As demonstrated in Supplementary Table 4, coumarin and shikimic acid showed the least binding affinity towards AR proteins. Although coumarin and shikimic acid establish a greater number of $\mathrm{H}$-bond interactions than the eugenol-AR system (Figures 6(a), 6(b), and 6(e)), the better binding affinity of eugenol to AR than coumarin and shikimic suggests that other nonpolar interactions also play a critical role apart from $\mathrm{H}$-bonding interaction towards the binding of the ligand with the receptor. Like the TNF-bonded system, in the AR-standard complex, alrestatin establishes five $\mathrm{H}$-bond interactions in the same binding cavity which was observed for all the studied compounds (Supplementary Figures $1 \mathrm{~B}$ and 1D). Interestingly, a similar pattern of interaction has also been observed in the experimentally determined cocrystalized conformation of alrestatin in AR (PDB ID: 1AZ1). These findings further support the reliability of our docking results.

\section{Discussion}

Viola odorata is a medicinal herb used for centuries. This herb in the TCM system treats diabetes, bronchitis, and many kidney and liver disorders; however, the mechanism of action of this herb is still not known like other TCMs. So, in this study, an in silico analysis was done to find out the antimetabolic activity of Viola odorata. Thus, various interventions were adopted to conduct the in silico study of Viola odorata; these include the study of drug targets and building the protein-protein interaction network and pathways which were combined systematically to find out the potential mode of action. As a result of the protein target investigation, a total of 119 targets were achieved. These protein targets were analyzed by GO enrichment analysis, which further proved that Viola odorata had antimetabolic activity. According to pathway enrichment analysis, Viola odorata regulates many pathways in adjunct with a wide range of therapeutic modules.

According to literature, the active constituents of Viola odorata involved in the treatment of diabetes are aodoratine, rutin, limonene, coumarins, cycloviolacin, vanillic acid, eugenol, and stigmasterol [9-12]. These compounds exhibited their activity against different diseases in animal models. Aodoratine, in the form of chewed leaves, was used as an anticancer drug [14]. Coumarin is a potent antidiabetic agent, as it showed a decreased level of plasma glucose and increased level of insulin by activating insulin secretion via shutting $\mathrm{K}^{+}$channels and opening $\mathrm{Ca}^{++}$channels in rats [21]. Cycloviolacin O8, also known as nematocidal cyclotide, showed activity against prostate, breast, and ovarian cancer cell lines. Cycloviolacin showed anticancer activity due to its possible antiproliferative activity [22]. Eugenol has many uses as a pharmacological moiety. It is the main active constituent of Viola odorata. In an 5experiment, the fructosefed rats were tested for the level of T-Ch, LDL-Ch, TG, and HDL-Ch for the treatment of diabetes. Resultantly, diabetes was promisingly treated due to antioxidant and lipidlowering activity of eugenol [23]. The metabolic complications such as cardiovascular disease were also potentially treated by using the combination of aspirin and eugenol ester as compared to precursor aspirin due to lesser side effects 
TABLE 2: Action view of predicted, functional protein targets (related to diabetes) of Viola odorata retrieved via STITCH.

\begin{tabular}{|c|c|c|c|c|c|c|c|c|c|}
\hline Proteins & Activation & Inhibition & Binding & Phenotype & Catalysis & Posttranslational modification & Reaction & Expression & Score \\
\hline CCND1 & - & $\bullet$ & $\bullet$ & & & & - & $\bullet$ & 0.999 \\
\hline CCND3 & & $\bullet$ & $\bullet$ & & & & $\bullet$ & & 0.999 \\
\hline CDKN2A & $\bullet$ & $\bullet$ & $\bullet$ & & $\bullet$ & $\bullet$ & & & 0.999 \\
\hline RB1 & $\bullet$ & $\bullet$ & $\bullet$ & & $\bullet$ & $\bullet$ & $\bullet$ & & 0.999 \\
\hline CDKN1B & $\bullet$ & $\bullet$ & $\bullet$ & & $\bullet$ & $\bullet$ & $\bullet$ & $\bullet$ & 0.999 \\
\hline CDKN1A & $\bullet$ & $\bullet$ & $\bullet$ & & $\bullet$ & $\bullet$ & $\bullet$ & $\bullet$ & 0.999 \\
\hline IL6R & $\bullet$ & & $\bullet$ & & & & & & 0.999 \\
\hline BCL2L11 & & & $\bullet$ & & & & & $\bullet$ & 0.999 \\
\hline CCND2 & $\bullet$ & $\bullet$ & $\bullet$ & & & & $\bullet$ & & 0.999 \\
\hline CDKN2B & $\bullet$ & $\bullet$ & $\bullet$ & & $\bullet$ & $\bullet$ & & & 0.999 \\
\hline Isoproterenol & $\bullet$ & $\bullet$ & $\bullet$ & & $\bullet$ & & & $\bullet$ & 0.999 \\
\hline $\mathrm{BAD}$ & & $\bullet$ & $\bullet$ & & & & $\bullet$ & & 0.999 \\
\hline BECN1 & & & $\bullet$ & & & & & $\bullet$ & 0.999 \\
\hline CDKN2C & $\bullet$ & • & $\bullet$ & & $\bullet$ & $\bullet$ & & & 0.999 \\
\hline TP53 & $\bullet$ & $\bullet$ & $\bullet$ & & & $\bullet$ & $\bullet$ & $\bullet$ & 0.999 \\
\hline BIK & & & $\bullet$ & & & & - & & 0.999 \\
\hline IL6ST & $\bullet$ & $\bullet$ & $\bullet$ & & & & & & 0.999 \\
\hline Progesterone & $\bullet$ & $\bullet$ & $\bullet$ & & - & & & $\bullet$ & 0.999 \\
\hline BAX & $\bullet$ & $\bullet$ & $\bullet$ & & & $\bullet$ & & $\bullet$ & 0.999 \\
\hline Mifepristone & - & $\bullet$ & • & & $\bullet$ & & & $\bullet$ & 0.999 \\
\hline
\end{tabular}

[40]. It was reported that eugenol had potent anticancer activity against MCF-7 breast cancer cells through apoptosis and inhibition of cell division. The other activities involved are the reduction in glutathione level and an increase in the lipid peroxidation level [41]. Additionally, eugenol has strong activity against aflatoxin-induced lipid peroxidation. Aflatoxins, a carcinogenic agent, are produced as a secondary metabolite of Aspergillus flavus. The plant showed its effect by decreasing NADPH-dependent cytochrome $\mathrm{C}$ reductase [42]. The antidiabetic and antioxidative activity and insulin sensitivity of eugenol were studied by Al-Trad et al., which were reported in diabetic rats. Along with other mechanisms, the insulin sensitivity can be restored by the activation of the GLUT4-AMPK signaling pathway [43]. The antihyperglycemic activity was discussed in a study in which the liver marker enzymes, the enzymes of carbohydrate metabolism, i.e., creatinine kinase and urea nitrogen, were assessed after administration of eugenol to diabetic rats. The rats showed an improved marker value as compared to normal, in addition to an improvement in weight and hepatic values and reduced adverse effects [44]. Another constituent, limonene, has strong antimetabolic activity as an anticancerous and antidiabetic agent. d-Limonene showed activity as an antidiabetic agent by reducing the level of plasma glucose, glycosylated hemoglobin levels, and activity of gluconeogenic enzymes and increasing glucokinase activity and liver glycogen level [45]. It was confirmed that d-limonene not only decreases the white and brown adipocytes, serum triglycerides, and glucose levels in the blood of the obese mice but also inhibits lipid accumulation in the liver. It also reduces HDL and LDL via activation of the peroxisome proliferatoractivated receptor involving $\alpha$-signaling and inhibition of liver X-receptor by $\beta$-signaling [46]. Another research group reported the treatment of diabetes associated with nonalcoholic fatty liver disease by d-limonene [47]. In addition, the hypocholesterolemic effect of rutin was described, stating that rutin, in combination with lovastatin, decreases the level of LDL and HDL and liver enzymes [48].

In PPIN, the main hubs such as TP53, BCL2, CDKN1A, 1L6, CCND1, RB1, and CDNK2A have already been reported to be related to diabetes. TP53 is also known as a tumor suppressor gene. The downregulation of TP53 causes the development of cancer in humans. Downregulation and overexpression of TP53 in response to any external pressure may be associated with diabetes such as obesity and insulin resistance. In humans, the common TP53 polymorph expresses at codon 72 , which produces protein variants R72 and P72. Thus, the R72TP53 is more active in fat deposition, nonalcoholic fatty liver disease, adipose tissue inflammation, and insulin resistance as compared to P721P53 [48]. According to another study, TP53 was not only found at the short arm of chromosome 17 but also linked with bladder cancer in humans; thus, it could be involved in tumorigenesis and can be used as a progression marker for the diagnostic purpose [49]. BCL2, another neurotrophic factor, is involved in regulating the primary function of the cell, viz., apoptosis. Any dysfunction in apoptosis causes various diseases, such as diabetes and cancer [50]. CDKN1A boosts up the cell division and cell cycle progression when targeted by an increased number of microRNA-93 in polycystic ovarian syndrome. The increased expression of microRNA-93 promotes cell division from the G1 to $\mathrm{S}$ phase. When the CDKN1A was eliminated from the granulosa cell, it promotes cell growth and cell cycle development from the previous phase. The 


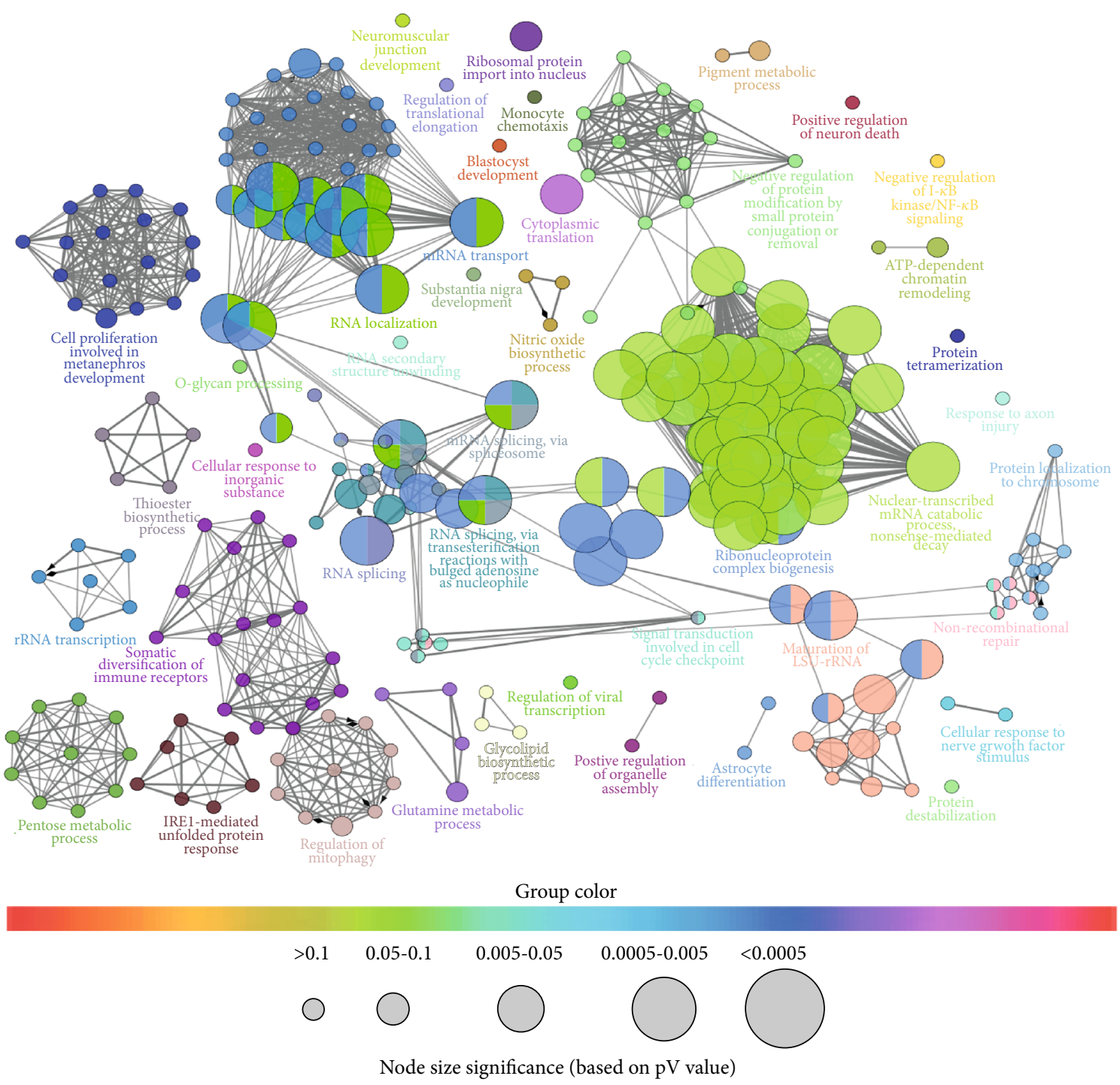

FIGURE 4: ClueGO-mediated retrieval of functionally grouped networks for the identified targets of Viola odorata. Each group is represented by the most significant term. The terms are represented by the nodes. The size of the nodes is associated with the significance of term enrichment. There is partial overlapping of the groups with similar functions.

reintroduction of $\mathrm{CDK} 1 \mathrm{~A}$ reverses its function, and instead of progression, it suppresses the growth and development of the cell. When insulin concentration is high in the cell, the microRNA-93 increases the sensitivity of the cell, which further activates KGN cell division and downregulation of CDK1A [51]. Interleukin-6 is a primary metabolic regulator and cytokine that plays a vital role in obesity and insulin resistance. It is a well-known fact that mitochondrial deformity is the reason for causing diabetes. Cells treated with IL6 have shown lesser lipids and a high level of glucose release rate; further, it also diminishes the ATP production and membrane potential and increases reactive oxygen species. Thus, it gives lipolytic effect and causes mitochondrial deformation, but it does not affect insulin sensitivity [52]. IL6 plays a crucial role in insulin resistance and obesity. The level of IL6 is increased in obese persons, revealing that IL6 is the major contributor to insulin resistance in diabetic conditions [53]. CCND1 is also known as cyclin D1 present throughout the cell cycle and involved in insulin signaling [54]. Rb1 is the active compound reported to induce adipogenesis and expression of peroxisome proliferator-activated receptor gamma. The PPARgamma has an antagonist called GW9662 that blocks Rb1-induced 3T3-L1 differentiation. The treatment results in decreased expression of miR-27b and pri-miR-27b. The antagonist of PPAR reduces the inhibitory effect of $\mathrm{Rb} 1$ on both $\mathrm{miR}-27 \mathrm{~b}$ and pri-miR27b. Thus, Rb1 acts through PPAR to decrease the expression of mir-27b and mature mir-27b that progresses adipogenesis and PPAR expression; thus, Rb1 has antidiabetic activity [55]. CDNK2A has already been documented to be associated with cancer and diabetes. It is a genomic locus that contains important genes for protein coding. The point at which antisense IncRNA is encoded is called ANRIL. Diabetes is induced when ANRIL gets polymorphed [56]. When the nutrition load increases, 


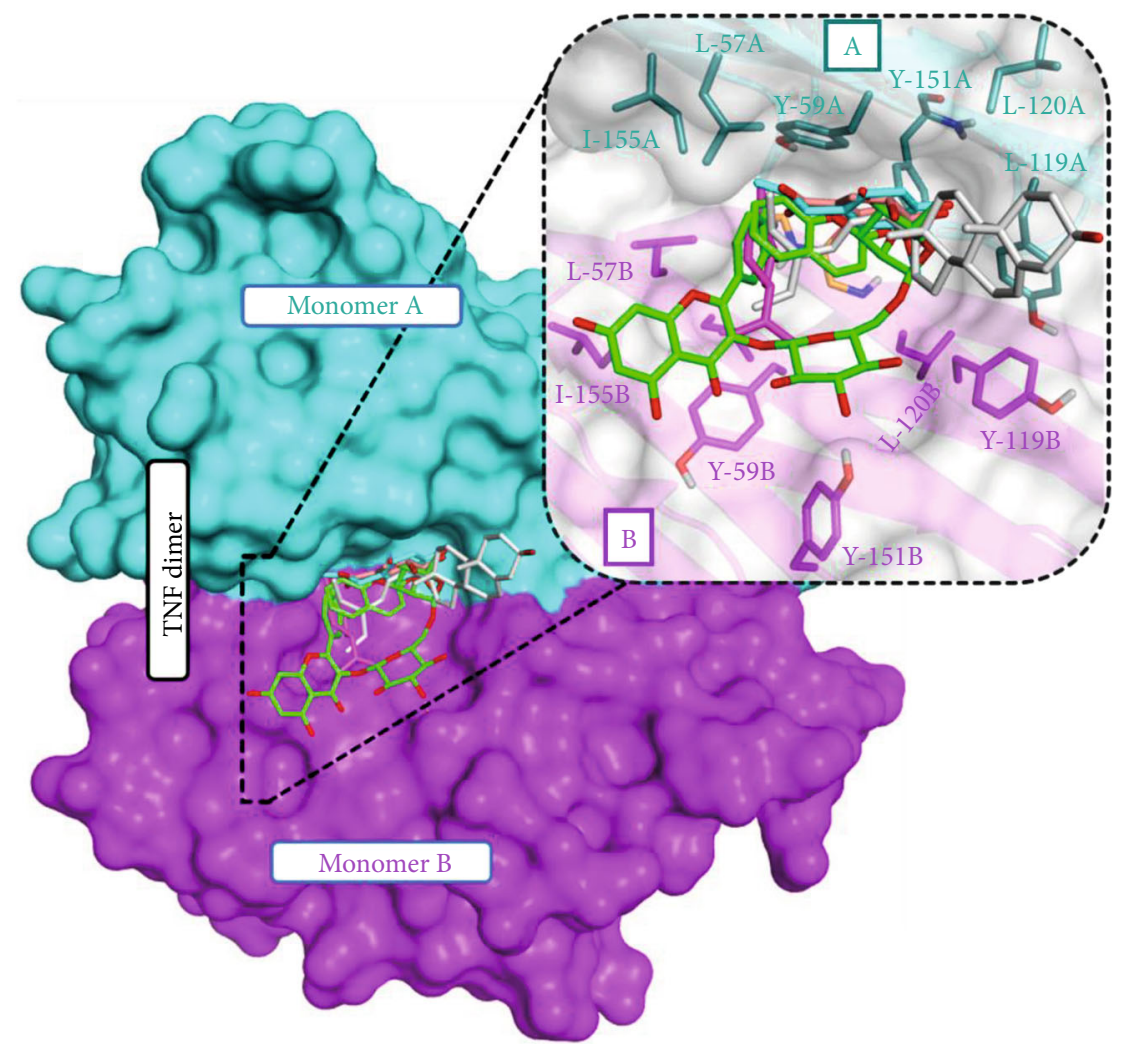

(a)

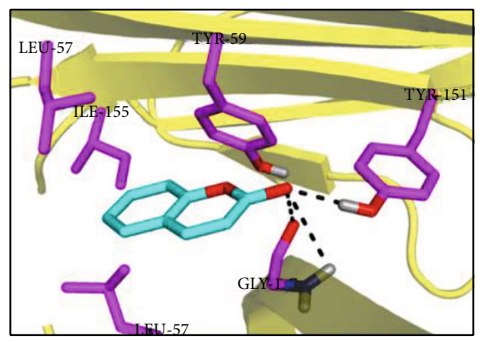

(b)

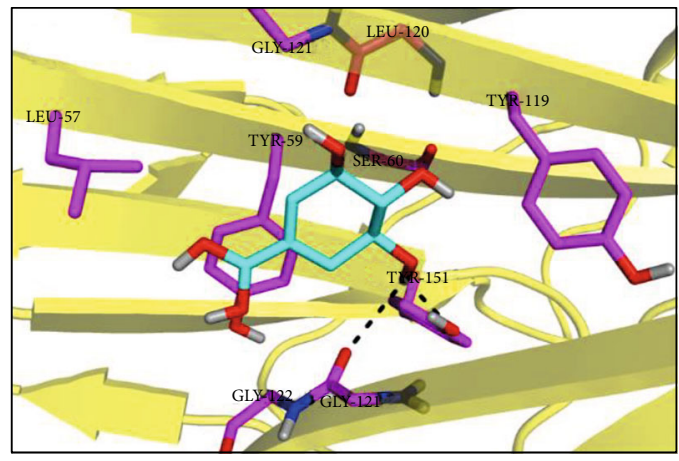

(e)

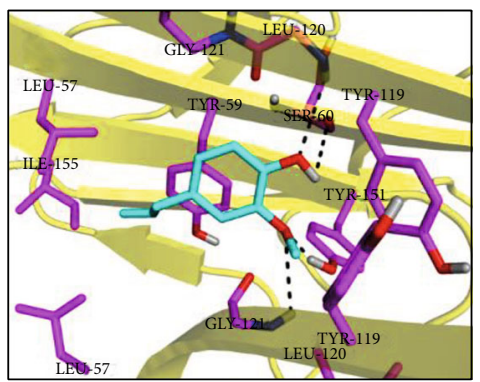

(c)

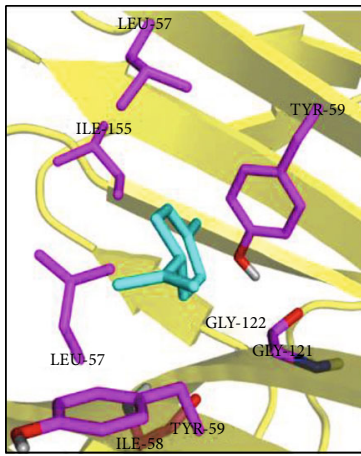

(f)

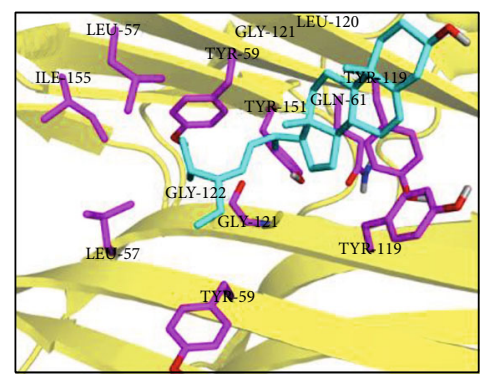

(d)

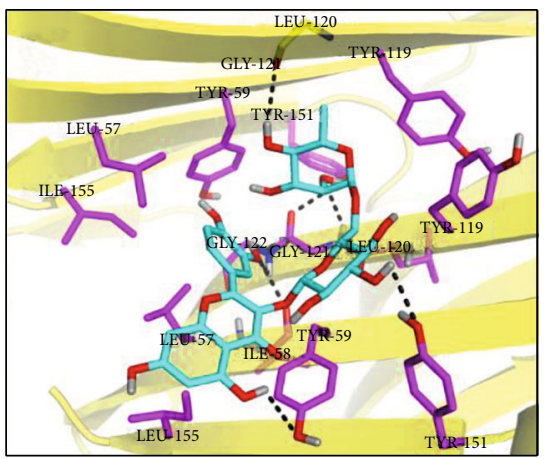

(g)

FIGURE 5: Obtained binding modes of ligands in the active site of tumor necrosis factor (TNF). (a) Docking conformations of the selected compounds bonded to the TNF dimer: (b) coumarin-TNF, (c) eugenol-TNF, (d) stigmasterol-TNF, (e) shikimic acid-TNF, (f) limoneneTNF, and (g) rutin-TNF.

insulin secretion decreases, leading to the development of diabetes type 2 . It is mainly caused by postprandial glucose regulation (PGR).
Pathway enrichment analysis reveals that most of the achieved GO terms have linkage with the diabetes. Nonetheless, three GO terms, i.e., glutamine metabolic process (GO: 


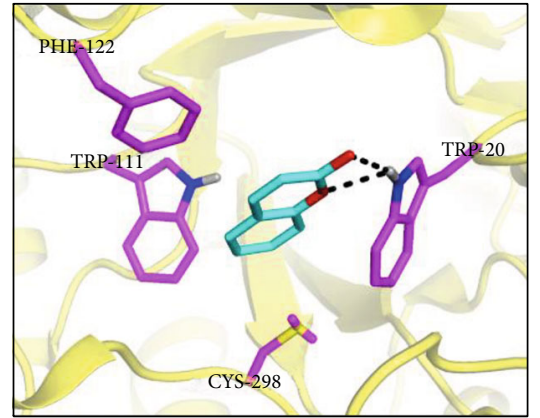

(a)

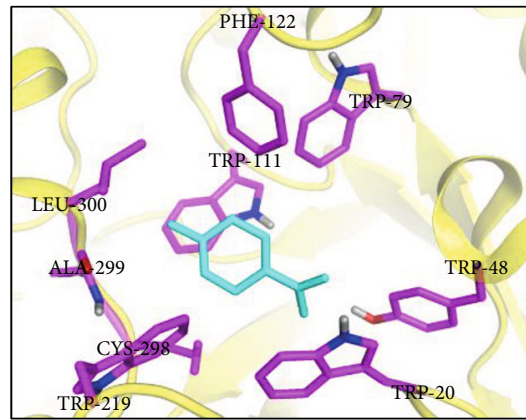

(c)

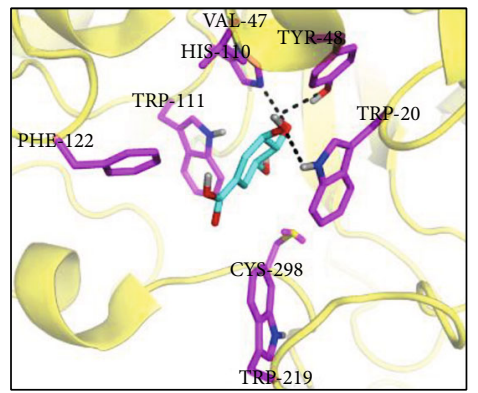

(e)

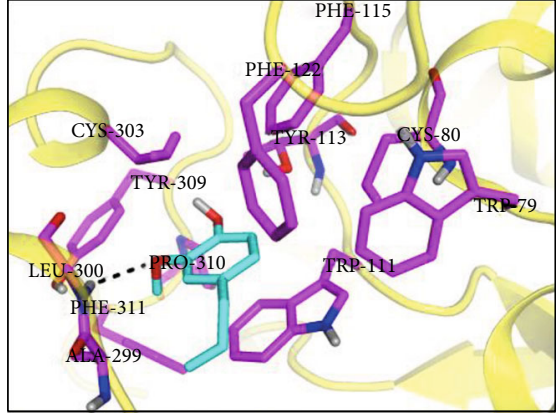

(b)

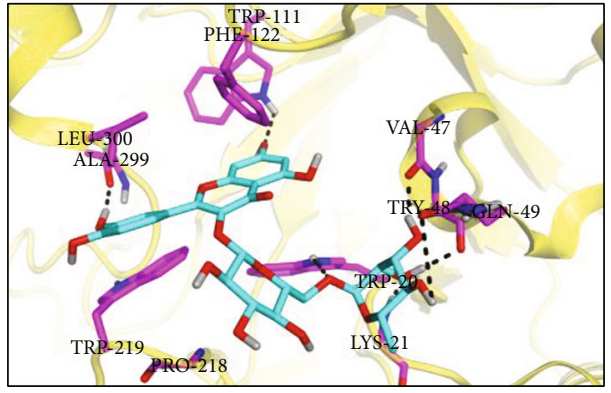

(d)

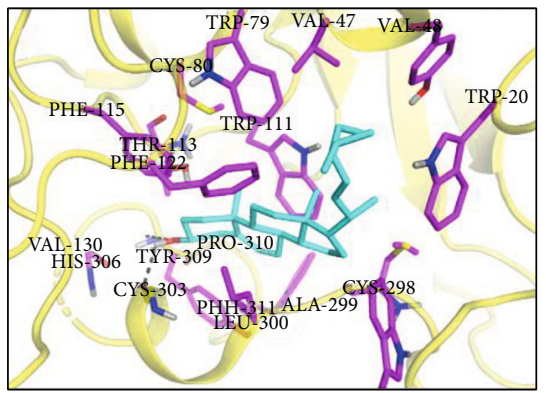

(f)

Figure 6: Obtained binding modes of ligands in the active site of aldose reductase (AR). (a) Docking conformations of the selected compounds bonded to AR: (b) coumarin-AR, (c) eugenol-AR, (d) stigmasterol-AR, (e) shikimic acid-AR, (f) limonene-AR, and (g) rutin-AR.

0006536), IRE1-mediated unfolded protein response (GO: 0006986), and pentose metabolic process (GO: 0019321), are related to the pathogenesis of diabetes. The glutamine metabolic process represents the reactions and pathways involving the metabolism of glutamates. The diabetesrelated conditions related to this GO term are hyperinsulinemia, hyperammonemia, and hypoglycemia [57]. Olivopontocerebellar atrophy is a common disease related to ataxia and Parkinson's-like diseases. The neurodegenerative process is linked with GDH (glycerate dehydrogenase) deficiency [58]. The regulation of diabetes is caused by genes including ASNS, CAD, GMPS, and PHGDH. Glutamate dehydrogenase secretes insulin from $\beta$-cells of the pancreas by activation of GDH via leucine [59]. When glutamate dehydrogenase enters the cell, glucose metabolism is activated via glucokinase resulting in the blockade of the $\mathrm{K}^{+}$channel. This phenomenon is known as leucine-sensitive hypoglycemia [60]. On the other hand, diabetes is also caused by endoplasmic reticulum (ER) stress, which is caused by misfolding of proteins [61]. These diseases include obesity and type 2 diabetes mellitus. Other diseases caused by ER stress are atherosclerosis, cardiovascular disease, stroke, ischemia, NAFLD, alcoholic fatty liver disease, steatosis, and cirrhosis. Thus, the treatment of diabetes could be associated with the unfolded protein response. The IRE1-mediated unfolded protein response is a process that leads to a changed state or activity of a cell or an organism in the context of enzyme production/secretion or gene expression. IRE1 is the first and foremost signaling entity of unfolded protein response. There are two forms of IRE1, i.e., IRE $1 \alpha$ and IRE1 $\beta$. IRE1 $\alpha$ is involved in signaling of unfolded protein response, and the IRE1 $\beta$ mechanism is still not clear. The genes involved in an unfolded protein response are ARFGAP1, SEC61A1, and TPP1. Under normal condition, when endoplasmic reticulum stress is not implied, IRE1 $\alpha$ regulates the glucose protein [62]. When stress is applied by ER, IRE1 $\alpha$ forms a homodimer and releases GRP78 which results in the interaction of the unfolded protein with ER resulting in obesity [63]. The unfolded protein response is an adaptive process that responds to metabolic stress, oxidative stress, and also 
inflammatory signaling pathways. For treatment purposes, the signaling of unfolded protein response is needed to be improved [64].

Pentose is a five-carbon sugar. The pentose metabolic process deals with the chemical reactions or pathways involving pentose. This pathway comprises two parts: an oxidative part and the nonoxidative part. The oxidative part is irreversible and allows the reduction of NADP to NADPH during the conversion of glucose-6-phosphate to pentose phosphate. While the nonoxidative part is reversible and interlinks the pentose phosphate to glycolytic intermediates [65], the genes found to be involved in the regulation of this pathway are DCXR, PGD, and PHGDH. The decreased level of pentose phosphate increases the occurrence of diabetes, likely due to the deficiency of G6PDH and RPI associated with sickle cell anemia [66]. It is also a documented fact that glucose6-phosphate dehydrogenase is the main rate-limiting enzyme involved in the pentose phosphate pathway. It has a prominent function in controlling vascular functions and causing cardiovascular risks along with obesity and dyslipidemia diabetes. In hyperglycemia and obesity, the G6PD expression is increased, leading to the development of insulin resistance [67].

This study explains various mechanisms involved in the treatment of diabetes by using Viola odorata and explains that this plant enriches the identified target genes that are involved in the treatment of metabolic faults such as insulin resistance. In short, this study has evaluated the therapeutic mode of Viola odorata in diabetes in a preclinical manner, which provides a base in the field of evidence-based medicines.

\section{Conclusion}

In this study, the mechanism underlying the antidiabetic effects of Viola odorata is promisingly identified by network pharmacology and confirmed by molecular docking. The findings revealed that several constituents of Viola odorata such as rutin, eugenol, vanillic acid, stigmasterol, and limonene have an association with insulin activity, which suggests that Viola odorata has the potential to manage diabetes. The overall effect could be additive based on the concept of multichemical-multitarget. The findings of this in silico study have helped to predict the molecular mechanism of Viola odorata and provided a piece of confirmation of its clinical use against diabetes. Furthermore, molecular docking studies identified rutin, stigmasterol, and eugenol as the most potent TNF and AR inhibitors among our selected compounds when compared to their corresponding standard inhibitors quercetin and alrestatin, respectively. Graphical analysis has shown that the ligands capable of establishing various $\mathrm{H}$ bonds as well as hydrophobic and van der Walls interactions are more strongly bonded in their corresponding complexes.

\section{Data Availability}

The supporting data has been provided as a supplementary file.

\section{Conflicts of Interest}

The authors declare that there is no conflict of interests.

\section{Acknowledgments}

This manuscript is a part of the project that was supported by the Deanship of Graduate Studies and Research (DGSR), Ajman University (grant no. 2019-IRG-PH-1). The authors, therefore, gratefully acknowledge the DGSR technical and financial support.

\section{Supplementary Materials}

The chemical constituents of Viola odorata and their protein targets are available as Supplementary Tables 1 and 2. Supplementary Table 3 presents the Gene Ontology terms and their related genes retrieved through ClueGO. The Surflex score of docked ligands in the active site of tumor necrosis factor (TNF) and aldose reductase (AR) is given in Supplementary Table 4. Supplementary Figure 1 describes the obtained binding modes of standard compounds quercetin and alrestatin bonded to their target proteins TNF and AR, respectively. (Supplementary Materials)

\section{References}

[1] M. H. Faulds and K. Dahlman-Wright, "Metabolic diseases and cancer risk," Current Opinion in Oncology, vol. 24, no. 1, pp. 58-61, 2012.

[2] P. Björntorp, "Body fat distribution, insulin resistance, and metabolic diseases," Nutrition, vol. 13, no. 9, pp. 795-803, 1997.

[3] A. K. Singh and J. A. Kari, "Metabolic syndrome and chronic kidney disease," Current Opinion in Nephrology and Hypertension, vol. 22, no. 2, pp. 198-203, 2013.

[4] C. A. Peralta, M. Kurella, J. C. Lo, and G. M. Chertow, "The metabolic syndrome and chronic kidney disease," Current Opinion in Nephrology and Hypertension, vol. 15, no. 4, pp. 361-365, 2006.

[5] Z. Azari, Z. Kherullahi, F. Mohammadghasemi, M. Aghajany Nasab, F. Hoseini, and R. Gazor, "Effect of the aqueous and hydro-alcoholic extracts of Viola odorata L. on biochemical and histologic liver parameters in diabetic Wistar rats," Anatomical Sciences Journal, vol. 17, no. 1, pp. 21-32, 2020.

[6] A. Vishal, K. Parveen, S. Pooja, N. Kannappan, and S. Kumar, "Diuretic, laxative and toxicity studies of Viola odorata aerial parts,” Pharmacology Online, vol. 1, pp. 739-748, 2009.

[7] H. Alipanah, M. R. Bigdeli, and M. A. Esmaeilib, "Inhibitory effect of Viola odorata extract on tumor growth and metastasis in 4T1 breast cancer model Iran J," Pharmaceutical Research, vol. 17, no. 1, pp. 276-291, 2018.

[8] M. Pranting, C. Loov, R. Burman, U. Goransson, and D. I. Andersson, "The cyclotide cycloviolacin O2 from Viola odorata has potent bactericidal activity against Gram-negative bacteria," J Antimicrob Chemother, vol. 65, no. 9, pp. 1964-1971, 2010.

[9] M. Kibble, N. Saarinen, J. Tang, K. Wennerberg, S. Mäkelä, and T. Aittokallio, "Network pharmacology applications to map the unexplored target space and therapeutic potential of 
natural products," Natural Product Reports, vol. 32, no. 8, pp. 1249-1266, 2015.

[10] J. Gu, H. Zhang, L. Chen, S. Xu, G. Yuan, and X. Xu, "Drugtarget network and polypharmacology studies of a Traditional Chinese Medicine for type II diabetes mellitus," Computational Biology and Chemistry, vol. 35, no. 5, pp. 293297, 2011.

[11] J. Yan, Y. Wang, S. J. Luo, and Y. J. Qiao, "TCM grammar systems: an approach to aid the interpretation of the molecular interactions in Chinese herbal medicine," Journal of Ethnopharmacology, vol. 137, no. 1, pp. 77-84, 2011.

[12] G. B. Zhang, Q. Y. Li, Q. L. Chen, and S. B. Su, "Network pharmacology: a new approach for Chinese herbal medicine research," Evidence-based Complementary and Alternative Medicine, vol. 2013, Article ID 621423, 9 pages, 2013.

[13] D. Cheng, G. Murtaza, S. Ma et al., "In silico prediction of the anti-depression mechanism of a herbal formula (Tiansi liquid) containing Morinda officinalis and Cuscuta chinensis," Molecules, vol. 22, no. 10, p. 1614, 2017.

[14] D. Chandra, G. Kohli, K. Prasad et al., "Phytochemical and ethnomedicinal uses of family Violaceae," Current Research in Chemistry, vol. 7, no. 2, pp. 44-52, 2015.

[15] E. Svangård, U. Göransson, D. Smith et al., "Primary and 3-D modelled structures of two cyclotides from Viola odorata," Phytochemistry, vol. 64, no. 1, pp. 135-142, 2003.

[16] D. C. Ireland, M. L. Colgrave, P. Nguyencong, N. L. Daly, and D. J. Craik, "Discovery and characterization of a linear cyclotide from Viola odorata: implications for the processing of circular proteins," Journal of Molecular Biology, vol. 357, no. 5, pp. 1522-1535, 2006.

[17] P. Mittal, V. Gupta, M. Goswami, N. Thakur, and P. Bansal, "Phytochemical and pharmacological potential of Viola odorata," International Journal of Pharmacognosy (IJP), vol. 4, p. 693, 2015.

[18] N. Muhammad, M. Saeed, A. Aleem, and H. Khan, "Ethnomedicinal, phytochemical and pharmacological profile of genus Viola," Phytopharmacol, vol. 3, no. 1, pp. 214-226, 2012.

[19] N. Kannappan, A. Diwan, P. Saini, S. Singh, V. Antil, and P. Kumar, "Evaluation of the analgesic activity of Viola odorata aerial parts in rats," Journal of Natural Pharmaceuticals, vol. 2, no. 1, p. 24, 2011.

[20] B. M. Jaber and S. F. Jasim, "Phytochemical study of stigmasterol and $\beta$-sitosterol in Viola odorata plant cultivated in Iraq," Iraqi Journal of Biotechnology, vol. 13, no. 2, pp. 86-94, 2014.

[21] L. Pari and N. Rajarajeswari, "Efficacy of coumarin on hepatic key enzymes of glucose metabolism in chemical induced type 2 diabetic rats," Chemico-Biological Interactions, vol. 181, no. 3, pp. 292-296, 2009.

[22] N. C. Parsley, C. L. Kirkpatrick, C. M. Crittenden et al., "PepSAVI-MS reveals anticancer and antifungal cycloviolacins in Viola odorata," Phytochemistry, vol. 152, pp. 61-70, 2018.

[23] S. Y. Al-Okbi, D. A. Mohamed, T. E. Hamed, and A. E. Edris, "Protective effect of clove oil and eugenol microemulsions on fatty liver and dyslipidemia as components of metabolic syndrome," Journal of Medicinal Food, vol. 17, no. 7, pp. 764$771,2014$.

[24] M. Clark, R. D. Cramer, and N. Van Opdenbosch, "Validation of the general purpose Tripos 5.2 force field," Journal of Computational Chemistry, vol. 10, no. 8, pp. 982-1012, 1989.

[25] M. M. He, "Small-molecule inhibition of TNF-," Science, vol. 310, no. 5750, pp. 1022-1025, 2005.
[26] P. Antony and R. Vijayan, "Identification of novel aldose reductase inhibitors from spices: a molecular docking and simulation study," PLoS One, vol. 10, no. 9, article e0138186, 2015.

[27] T. A. Chohan, J. J. Chen, H. Y. Qian, Y. L. Pan, and J. Z. Chen, "Molecular modeling studies to characterize N-phenylpyrimidin-2-amine selectivity for CDK2 and CDK4 through 3DQSAR and molecular dynamics simulations," Molecular BioSystems, vol. 12, no. 4, pp. 1250-1268, 2016.

[28] K. Rehman, T. A. Chohan, I. Waheed, Z. Gilani, and M. S. H. Akash, "Taxifolin prevents postprandial hyperglycemia by regulating the activity of $\alpha$-amylase: evidence from an in vivo and in silico studies," Journal of Cellular Biochemistry, vol. 120, no. 1, pp. 425-438, 2018.

[29] W. Welch, J. Ruppert, and A. N. Jain, "Hammerhead: fast, fully automated docking of flexible ligands to protein binding sites," Chemistry \& Biology, vol. 3, no. 6, pp. 449-462, 1996.

[30] H. Yang, T. Yang, C. Heng et al., "Quercetin improves nonalcoholic fatty liver by ameliorating inflammation, oxidative stress, and lipid metabolism in $\mathrm{db} / \mathrm{db}$ mice," Phytotherapy Research, vol. 33, no. 12, pp. 3140-3152, 2019.

[31] Y. Liu, C. Yu, K. Ji et al., "Quercetin reduces TNF- $\alpha$-induced mesangial cell proliferation and inhibits PTX3 production: involvement of NF- $\kappa \mathrm{B}$ signaling pathway," Phytotherapy Research, vol. 33, no. 9, pp. 2401-2408, 2019.

[32] Z. Yuan, J. Min, Y. Zhao et al., "Quercetin rescued TNF-alphainduced impairments in bone marrow-derived mesenchymal stem cell osteogenesis and improved osteoporosis in rats," American Journal of Translational Research, vol. 10, no. 12, pp. 4313-4321, 2018.

[33] Z. Weng, B. Zhang, S. Asadi et al., "Quercetin is more effective than cromolyn in blocking human mast cell cytokine release and inhibits contact dermatitis and photosensitivity in humans," PLoS One, vol. 7, no. 3, article e33805, 2012.

[34] S.-C. Cheng, W.-C. Huang, J.-H. S. Pang, Y.-H. Wu, and C.Y. Cheng, "Quercetin inhibits the production of IL- $1 \beta$-induced inflammatory cytokines and chemokines in ARPE- 19 cells via the MAPK and NF- $\kappa$ B signaling pathways," International Journal of Molecular Sciences, vol. 20, no. 12, p. 2957, 2019.

[35] L. T. Chylack Jr., H. F. Henriques III, H. M. Cheng, and W. H. Tung, "Efficacy of alrestatin, an aldose reductase inhibitor, in human diabetic and nondiabetic lenses," Ophthalmology, vol. 86, no. 9, pp. 1579-1585, 1979.

[36] J. A. Jedziniak, L. T. Chylack, H. M. Cheng, M. K. Gillis, A. A. Kalustian, and W. H. Tung, "The sorbitol pathway in the human lens: aldose reductase and polyol dehydrogenase," Investigative Ophthalmology \& Visual Science, vol. 20, no. 3, pp. 314-326, 1981.

[37] A. S. Grewal, S. Bhardwaj, D. Pandita, V. Lather, and B. S. Sekhon, "Updates on aldose reductase inhibitors for management of diabetic complications and non-diabetic diseases," Mini Reviews in Medicinal Chemistry, vol. 16, no. 2, pp. 120 $162,2016$.

[38] A. N. Jain, "Surflex: fully automatic flexible molecular docking using a molecular similarity-based search engine," Journal of Medicinal Chemistry, vol. 46, no. 4, pp. 499-511, 2003.

[39] G. Melagraki, E. Ntougkos, D. Papadopoulou et al., "In silico discovery of plant-origin natural product inhibitors of tumor necrosis factor (TNF) and receptor activator of NF- $\kappa \mathrm{B}$ ligand (RANKL)," Frontiers in Pharmacology, vol. 9, p. 800, 2018.

[40] Y. Shen, X. Liu, Y. Yang, J. Li, N. Ma, and B. Li, "In vivo and in vitro metabolism of aspirin eugenol ester in dog by liquid 
chromatography tandem mass spectrometry," Biomedical Chromatography, vol. 29, no. 1, pp. 129-137, 2015.

[41] N. Vidhya and S. N. Devaraj, "Induction of apoptosis by eugenol in human breast cancer cells," Indian Journal of Experimental Biology, vol. 49, no. 11, pp. 871-878, 2011.

[42] T. Jayashree and C. Subramanyam, "Antiaflatoxigenic activity of eugenol is due to inhibition of lipid peroxidation," Letters in Applied Microbiology, vol. 28, no. 3, pp. 179-183, 1999.

[43] B. Al-Trad, H. Alkhateeb, W. Alsmadi, and M. Al-Zoubi, "Eugenol ameliorates insulin resistance, oxidative stress and inflammation in high fat-diet/streptozotocin-induced diabetic rat," Life Sciences, vol. 216, pp. 183-188, 2019.

[44] S. Srinivasan, G. Sathish, M. Jayanthi, J. Muthukumaran, U. Muruganathan, and V. Ramachandran, "Ameliorating effect of eugenol on hyperglycemia by attenuating the key enzymes of glucose metabolism in streptozotocin-induced diabetic rats," Molecular and Cellular Biochemistry, vol. 385, no. 1-2, pp. 159-168, 2014.

[45] R. Murali and R. Saravanan, "Antidiabetic effect of d-limonene, a monoterpene in streptozotocin-induced diabetic rats," Biomedicine \& Preventive Nutrition, vol. 2, no. 4, pp. 269-275, 2012.

[46] L. Jing, Y. Zhang, S. Fan et al., "Preventive and ameliorating effects of citrus D-limonene on dyslipidemia and hyperglycemia in mice with high-fat diet-induced obesity," European Journal of Pharmacology, vol. 715, no. 1-3, pp. 46-55, 2013.

[47] J. V. A. Santiago, J. Jayachitra, M. Shenbagam, and N. Nalini, "Dietary d-limonene alleviates insulin resistance and oxidative stress-induced liver injury in high-fat diet and L-NAMEtreated rats," European Journal of Nutrition, vol. 51, no. 1, pp. 57-68, 2012.

[48] M. Lacroix, R. Riscal, G. Arena, L. K. Linares, and L. Le Cam, "Metabolic functions of the tumor suppressor p53: implications in normal physiology, metabolic disorders, and cancer," Molecular metabolism, vol. 33, pp. 2-22, 2020.

[49] P. Lianes, I. Orlow, Z. F. Zhang et al., "Altered patterns of MDM2 and TP53 expression in human bladder cancer," JNCI: Journal of the National Cancer Institute, vol. 86, no. 17, pp. 1325-1330, 1994.

[50] W. A. Siddiqui, A. Ahad, and H. Ahsan, "The mystery of BCL2 family: BCL-2 proteins and apoptosis: an update," Archives of Toxicology, vol. 89, no. 3, pp. 289-317, 2015.

[51] L. Jiang, J. Huang, L. Li et al., "MicroRNA-93 promotes ovarian granulosa cells proliferation through targeting CDKN1A in polycystic ovarian syndrome," The Journal of Clinical Endocrinology \& Metabolism, vol. 100, no. 5, pp. E729-E738, 2015.

[52] C. Ji, X. Chen, C. Gao et al., "IL-6 induces lipolysis and mitochondrial dysfunction, but does not affect insulin-mediated glucose transport in 3T3-L1 adipocytes," Journal of Bioenergetics and Biomembranes, vol. 43, no. 4, pp. 367-375, 2011.

[53] T. L. Allen and M. A. Febbraio, "IL6 as a mediator of insulin resistance: fat or fiction," Diabetologia, vol. 53, no. 3, pp. 399-402, 2010.

[54] Y. Lee, J. E. Dominy, Y. J. Choi et al., "Cyclin D1-Cdk4 controls glucose metabolism independently of cell cycle progression," Nature, vol. 510, no. 7506, pp. 547-551, 2014.

[55] L.-S. Chan, P. Yue, T.-W. Kok, M.-H. Keung, N.-K. Mak, and R. Wong, "Ginsenoside-Rb1 promotes adipogenesis through regulation of PPAR $\gamma$ and microRNA-27b," Hormone and Metabolic Research, vol. 44, no. 11, pp. 819-824, 2012.
[56] Y. Kong, C. H. Hsieh, and L. C. Alonso, “ANRIL: a lncRNA at the CDKN2A/B locus with roles in cancer and metabolic disease," Frontiers in Endocrinology, vol. 9, 2018.

[57] C. A. Stanley, Y. K. Lieu, B. Y. L. Hsu et al., "Hyperinsulinism and hyperammonemia in infants with regulatory mutations of the glutamate dehydrogenase gene," New England Journal of Medicine, vol. 338, no. 19, pp. 1352-1357, 1998.

[58] P. Andreas, F. Panayiotis, B. N. Anastasia, and P. Shashidharan, "Glutamate dehydrogenase deficiency in cerebellar degenerations: clinical, biochemical and molecular genetic aspects," Canadian Journal of Neurological Sciences, vol. 20, no. S3, pp. S109-S116, 1993.

[59] A. Kelly and C. A. Stanley, "Disorders of glutamate metabolism," Mental Retardation and Developmental Disabilities Research Reviews, vol. 7, no. 4, pp. 287-295, 2001.

[60] V. Cormier-Daire, N. Dagoneau, R. Nabbout et al., "A gene for pyridoxine-dependent epilepsy maps to chromosome 5q31," The American Journal of Human Genetics, vol. 67, no. 4, pp. 991-993, 2000.

[61] F. Engin, A. Yermalovich, T. Nguyen et al., "Restoration of the unfolded protein response in pancreatic $\beta$ cells protects mice against type 1 diabetes," Science translational medicine, vol. 5, no. 211, article 211ra156, 2013.

[62] A. Bertolotti, Y. Zhang, L. M. Hendershot, H. P. Harding, and D. Ron, "Dynamic interaction of BiP and ER stress transducers in the unfolded-protein response," Nature cell biology, vol. 2, no. 6, pp. 326-332, 2000.

[63] B. M. Gardner and P. Walter, "Unfolded proteins are Ire1activating ligands that directly induce the unfolded protein response," Science, vol. 333, no. 6051, pp. 1891-1894, 2011.

[64] S. Wang and R. J. Kaufman, "The impact of the unfolded protein response on human disease," The Journal of Cell Biology, vol. 197, no. 7, pp. 857-867, 2012.

[65] M. M. C. Wamelink, E. A. Struys, and C. Jakobs, "The biochemistry, metabolism and inherited defects of the pentose phosphate pathway: a review," Journal of Inherited Metabolic Disease, vol. 31, no. 6, pp. 703-717, 2008.

[66] A. Stincone, A. Prigione, T. Cramer et al., "The return of metabolism: biochemistry and physiology of the pentose phosphate pathway," Biological Reviews, vol. 90, no. 3, pp. 927-963, 2015.

[67] S. A. Gupte, "Targeting the pentose phosphate pathway in syndrome X-related cardiovascular complications," Drug Development Research, vol. 71, no. 3, pp. 161-167, 2010. 